\title{
Research on IMM-UKF Based Civilian Unmanned Aerial Vehicle Integrated Navigation Algorithm
}

\author{
Ming Liu \\ Airport College, Binzhou University, Binzhou 256600, China \\ hitswordben@126.com
}

\begin{abstract}
The main navigation method of Civilian Unmanned Aerial Vehicle (CUAV) is Global Positioning System (GPS) navigation, GPS navigation failure will make CUAV lost control, resulting in heavy losses. How to ensure the reliability and navigation precision of CUAV under the failure of GPS is a hot issue currently studied. In this paper, the self-developed Beidou satellite navigation system (BDS) by China is used to assist the Inertial Navigation System (INS) of CUAV navigation, when the GPS navigation is failure. The IMM-UKF method is used to overcome the uncertainties of statistical characteristics and the dispersion of filtering. The algorithm is verified by MATLAB software. The simulation results show that compared with the traditional EKF algorithm, this IMM-UKF algorithm can improve the navigation accuracy of the integrated navigation system and prevent the filter diverging. It can effectively solve the problem of navigational problems of CUAV under GPS failure.
\end{abstract}

Key words: CUAV; GPS; navigation; INS; BDS; Global Positioning System; IMM-UKF.

\section{INTRODUCTION}

The research of CUAV has made great progress in recent years, and its application field is also getting wider and wider. CUAV generally use the Micro-Electro Mechanical Systems \& Strapdown Inertial Navigation System (MEMS-SINS) and GPS combination as the navigation method and use the advantages of GPS long-time accuracy to correct the error drift of inertial navigation, so as to obtain good positioning accuracy [1]. However, civilian drones flying at low altitude, GPS signals vulnerable to intentional or unintentional interference or multipath error and other factors, GPS is not always available [2]. Once the GPS fails, accuracy and stability of the MEMS inertial device is still relatively low, resulting in reduced navigation accuracy. In more serious cases, the UAV may lose control and thus lose or crashed, resulting in significant economic losses and ground crew injuries. Therefore, it is of great significance to study the navigation of civil UAVs under the failure of GPS.

In the case of GPS failure, many scholars at home and abroad have proposed a variety of low altitude CUAV combination navigation methods, mainly INS/geomagnetic navigation, INS / terrain matching combination navigation [3, 4]. Navigation methods such as geomagnetic navigation and terrain matching navigation often have the advantage of working around the clock and obtaining high accuracy of information. However, since a large amount of geographic feature information data needs to be stored in advance, a large amount of data calculation is required in the navigation process and navigation computer requirements are relatively high, It is not suitable for smaller CUAV use.

China's Beidou satellite navigation system is a phased evolution of the satellite navigation system, designed to provide the Chinese army, civilian users to locate, group user management and precision timing services. Beidou satellite navigation system on December 27, 2012 announced the official operation, is for users in the Asia-Pacific region to provide independent navigation and positioning services. From the literature [5], Compass pseudo-range and carrier phase measurement accuracy with the GPS at the same level, pseudo-range measurement accuracy of about $33 \mathrm{~cm}$, carrier measurement accuracy of about $2 \mathrm{~mm}$; BDS pseudo-range point positioning accuracy is better 
than $6 \mathrm{~m}$, and the accuracy of elevation is better than $10 \mathrm{~m}$. The positioning accuracy is better than $1 \mathrm{~cm}$ when the BDS carrier phase difference is very short and the baseline is better than $3 \mathrm{~cm}$.

This article will study the failure of GPS using the BDS assistant the INS for CUAV navigation. The simulation study on the above BDS/INS integrated navigation system shows that the system can effectively improve the navigation accuracy and prevent the inertial navigation system from filtering divergence.

\section{IMM-UKF FILTERING ALGORITHM}

The complete IMM-UKF algorithm consists of four steps, namely input interaction, UKF filtering, model probability update and output combination [6].

\section{Input Interaction}

For a given state $x_{k-1}^{j}=x_{k-1 / k-1}^{j}$ and mixing probability $\mu_{k-1 / k-1}^{i / j}$, the initial estimated covariance is given by:

$$
\begin{gathered}
\hat{x}_{k-1 / k-1}^{0 j}=\sum_{i=1}^{r} \hat{x}_{k-1 / k-1}^{i} \mu_{k-1 / k-1}^{i / j} \\
P_{k-1 / k-1}^{0 j}=\sum_{i=1}^{r} \mu_{k-1 / k-1}^{i / j} \cdot\left\{P_{k-1 / k-1}^{i}+\left[\hat{x}_{k-1 / k-1}^{i}-\hat{x}_{k-1 / k-1}^{0 j}\right]\left[\hat{x}_{k-1 / k-1}^{i}-\hat{x}_{k-1 / k-1}^{0 j}\right]^{T}\right\}
\end{gathered}
$$

Where $P_{k-1}^{j}=P_{k-1 / k-1}^{j}$ is the corresponding covariance of the given state $x_{k-1}^{j}=x_{k-1 / k-1}^{j}$.

Model switching probability matrix is defined as:

$$
\pi_{i j}=p\left\{M_{k}^{j} / M_{k-1}^{i}\right\}=\left[\begin{array}{cccc}
\pi_{11} & \pi_{12} & \cdots & \pi_{1 j} \\
\pi_{21} & \pi_{22} & \cdots & \pi_{2 j} \\
\vdots & \vdots & \ddots & \vdots \\
\pi_{i 1} & \pi_{i 2} & \cdots & \pi_{i j}
\end{array}\right]
$$

Calculate the probability of mixing by $\pi_{i j}$, using the following formula to calculate the probability of Gaussian mixture:

$$
\mu_{k-1 / k-1}^{i / j}=\frac{1}{\bar{c}_{j}} \pi_{i j} \mu_{k-1}^{i}
$$

Where $\bar{c}_{j}=\sum_{i=1}^{r} \pi_{i j} \mu_{k-1}^{i}$ is the nonlinear factor.

\section{UKF Filtering}

1. Unscented transform

The Unscented transform produces $2 n+1$ Sigma vector $X$ and a weighted point W. At the moment $k-1$ of state estimation, the Sigma point is generated by the following equation. 


$$
\left\{\begin{array}{l}
X_{0, k-1}^{j}=\hat{x}_{k-1}^{0 j} \\
X_{i, k-1}^{j}=\hat{x}_{k-1}^{0 j}+\left(\sqrt{(n+\lambda) P_{k-1}^{0 j}}\right)_{i}^{T} \\
X_{i+n, k-1}^{j}=\hat{x}_{k-1}^{0 j}-\left(\sqrt{(n+\lambda) P_{k-1}^{0 j}}\right)_{i}^{T}
\end{array}\right.
$$

$W_{i}^{(m)}$ is the mean of points $i ; W_{i}^{(c)}$ is the covariance of points $i$.

$$
\begin{gathered}
W_{0}^{(m)}=\frac{\lambda}{n+\lambda} \\
W_{0}^{(c)}=W_{0}^{(m)}+\left(1-\alpha^{2}+\beta\right) \\
W_{i}^{(m)}=W_{i}^{(c)}=\frac{1}{2(n+\lambda)}
\end{gathered}
$$

2. Time update

$$
\begin{gathered}
\xi_{i, k / k-1}^{j}=f_{j}\left(X_{i, k-1}^{j}\right) \\
\hat{x}_{k / k-1}^{j}=\sum_{i=0}^{2 n} W_{i}^{(m)} \xi_{i, k / k-1}^{j} \\
P_{k / k-1}^{j}=\sum_{i=0}^{2 n} W_{i}^{(c)}\left[\xi_{i, k / k-1}^{j}-\hat{x}_{k / k-1}^{j}\right]\left[\xi_{i, k / k-1}^{j}-\hat{x}_{k / k-1}^{j}\right]^{T}+Q_{k-1}^{j} \\
Z_{i, k / k-1}^{j}=h\left(\xi_{i, k / k-1}^{j}\right) \\
\hat{Z}_{k / k-1}^{j}=\sum_{i=0}^{2 n} W_{i}^{(m)} Z_{i, k / k-1}^{j}
\end{gathered}
$$

3. Measurement update

$$
\begin{gathered}
P_{z z}^{j}=\sum_{i=0}^{2 n} W_{i}^{(c)}\left[Z_{i, k / k-1}^{j}-\hat{z}_{k / k-1}^{j}\right]\left[Z_{i, k / k-1}^{j}-\hat{z}_{k / k-1}^{j}\right]^{T}+R_{k}^{j} \\
P_{x z}^{j}=\sum_{i=0}^{2 n} W_{i}^{(c)}\left[\xi_{i, k / k-1}^{j}-\hat{x}_{k / k-1}^{j}\right]\left[Z_{i, k / k-1}^{j}-\hat{z}_{k / k-1}^{j}\right]^{T} \\
K_{k}^{j}=P_{x z}^{j}\left(P_{z z}^{j}\right)^{-1} \\
\hat{x}_{k / k}^{j}=\hat{x}_{k / k-1}^{j}+K_{k}^{j} v_{k}^{j} \\
v_{k}^{j}=z_{k}-z_{k / k-1}^{j}
\end{gathered}
$$




$$
P_{k / k}^{j}=P_{k / k-1}^{j}-K_{k}^{j} P_{z z}^{j}\left(K_{k}^{j}\right)^{T}
$$

\section{Model Probability Update}

$$
\begin{gathered}
\mu_{k}^{j}=\frac{1}{c} \Lambda_{k}^{j} \bar{C}_{j} \\
\Lambda_{k}^{j}=\left(\frac{1}{\sqrt{2 \pi\left|P_{z z}^{j}\right|}}\right)^{-\frac{1}{2}\left(v_{k}^{j}\right)^{T}\left(P_{z z}^{j}\right)^{-1} v_{k}^{j}}
\end{gathered}
$$

Where, $\mu_{k}^{j}$ is the model probability.

\section{Output Combination}

$$
\begin{gathered}
\hat{x}_{k / k}=\sum_{j=1}^{r} \hat{x}_{k / k}^{j} \mu_{k}^{j} \\
P_{k / k}=\sum_{j=1}^{r} \mu_{k}^{j}\left\{P_{k / k}^{j}+\left[\hat{x}_{k / k}^{j}-\hat{x}_{k / k}\right]\left[\hat{x}_{k / k}^{j}-\hat{x}_{k / k}\right]^{T}\right\}
\end{gathered}
$$

\section{MODEL AND IMM-UKF FILTER}

In this paper, the East-North-North coordinate system is selected as the navigation coordinate system. Supposing the change of sky direction velocity is zero, the error of gyroscope and accelerometer is reduced to the constant value plus white noise.

\section{State Equation}

1. Angle Error Equation

$$
\left\{\begin{aligned}
\dot{\phi}_{x}= & -\frac{\delta v_{y}}{R}+\left(\omega_{i e} \sin L+\frac{v_{x}}{R} \operatorname{tg} L\right) \phi_{y}-\left(\omega_{i e} \cos L+\frac{v_{x}}{R}\right) \phi_{z}+K_{g x}+w_{g x} \\
\dot{\phi}_{y}= & \frac{\delta v_{x}}{R}-\omega_{i e} \sin L \delta L-\left(\omega_{i e} \sin L+\frac{v_{x}}{R} \operatorname{tg} L\right) \phi_{x}-\frac{v_{y}}{R} \phi_{z}+K_{g y}+w_{g y} \\
\dot{\phi}_{z}= & \frac{\delta v_{x}}{R} \operatorname{tg} L+\left(\omega_{i e} \cos L+\frac{v_{x}}{R} \sec ^{2} L\right) \delta L+\left(\omega_{i e} \cos L+\frac{v_{x}}{R}\right) \phi_{x}+\frac{v_{y}}{R} \phi_{y} \\
& +K_{g z}+w_{g z}
\end{aligned}\right.
$$

2. Speed error equation 


$$
\left\{\begin{aligned}
\delta \dot{v}_{x}= & f_{y} \phi_{z}-f_{z} \phi_{y}+\frac{v_{y}}{R} \operatorname{tg} L \delta v_{x}+\left(2 \omega_{i e} \sin L+\frac{v_{x}}{R} \operatorname{tg} L\right) \delta v_{y} \\
& +\left(2 \omega_{i e} \cos L v_{y}+\frac{v_{x} v_{y}}{R} \sec ^{2} L\right) \delta L+K_{a x}+v_{a x} \\
\delta \dot{v}_{y}= & f_{z} \phi_{x}-f_{x} \phi_{z}-\left(2 \omega_{i e} \sin L+\frac{v_{x}}{R} \operatorname{tg} L\right) \delta v_{x} \\
& -\left(2 \omega_{i e} \cos L v_{x}+\frac{v_{x}^{2}}{R} \sec ^{2} L\right) \delta L+K_{a y}+v_{a y}
\end{aligned}\right.
$$

3. Position Error Equation

$$
\left\{\begin{array}{l}
\delta \dot{L}=\frac{\delta v_{y}}{R} \\
\delta \dot{\lambda}=\frac{\delta v_{x}}{R} \sec L+\frac{v_{x}}{R} \sec \operatorname{Ltg} L \delta L
\end{array}\right.
$$

The system state variables are angular error $\phi_{x}, \phi_{y}, \phi_{z}$, velocity error $\delta v_{x}, \delta v_{y}$, position error $\delta L, \delta \lambda$, and constant error of the gyroscope and accelerometer.

$$
X=\left[\phi_{x}, \phi_{y}, \phi_{z}, \delta v_{x}, \delta v_{y}, \delta L, \delta \lambda, K_{g x}, K_{g y}, K_{g z}, K_{a x}, K_{a y}\right]^{T}
$$

The system state equation is

$$
\dot{X}(t)=F(t) X(t)+G(t) w(t)
$$

\section{Observation Equation}

The position information output by BDS is a nonlinear equation, and the difference between the position provided by BDS and the corresponding output information of the inertial navigation system is taken as the observation value to obtain the nonlinear measurement equation as

$$
z(t)=H(X(t))+v(t)
$$

Which $v(t)$ is measure noise.

\section{SIMULATION RESULTS}

In order to verify the validity of IMM-UKF algorithm, MATLAB numerical verification is carried out and compared with the traditional EKF algorithm. CUAV flight route shown in Fig. 1. 


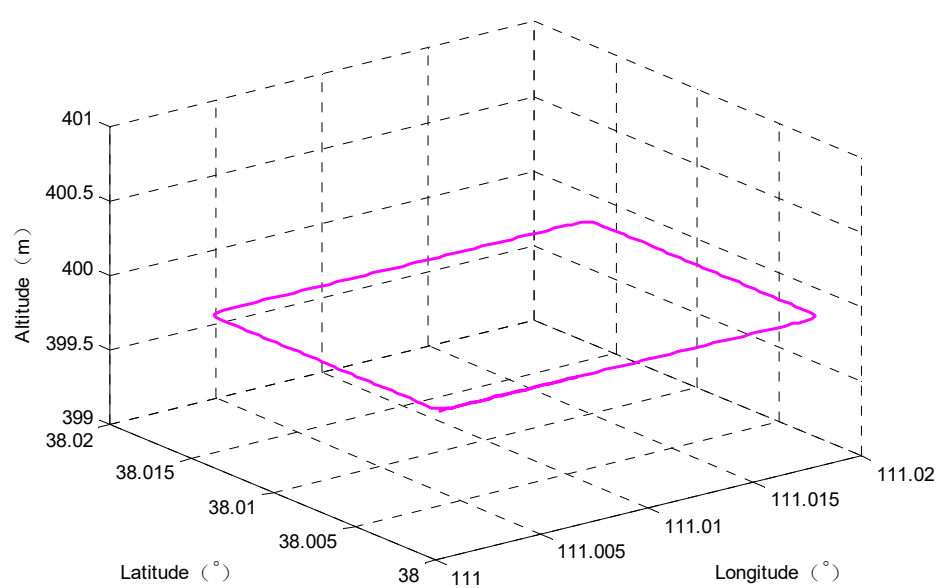

FIGURE 1. CUAV flight path

The initial velocity in the east and north directions is $16 \mathrm{~m} / \mathrm{s}$, the initial velocity error is $3 \mathrm{~m} / \mathrm{s}$, and the initial position errors are $1^{\circ}$ and $0.5^{\circ}$, respectively. Gyro constant error of $1^{\circ} / \mathrm{h}$, the accelerometer constant error is $10^{-3} \mathrm{~g}$. Simulation time is $2500 \mathrm{~s}$, filter cycle is $1 \mathrm{~s}$. The EKF algorithm and the IMM-UKF algorithm are respectively used to conduct the navigation and settlement of the BDS auxiliary inertial navigation system. East and north of the location error results shown in Fig. 2, Fig. 3.

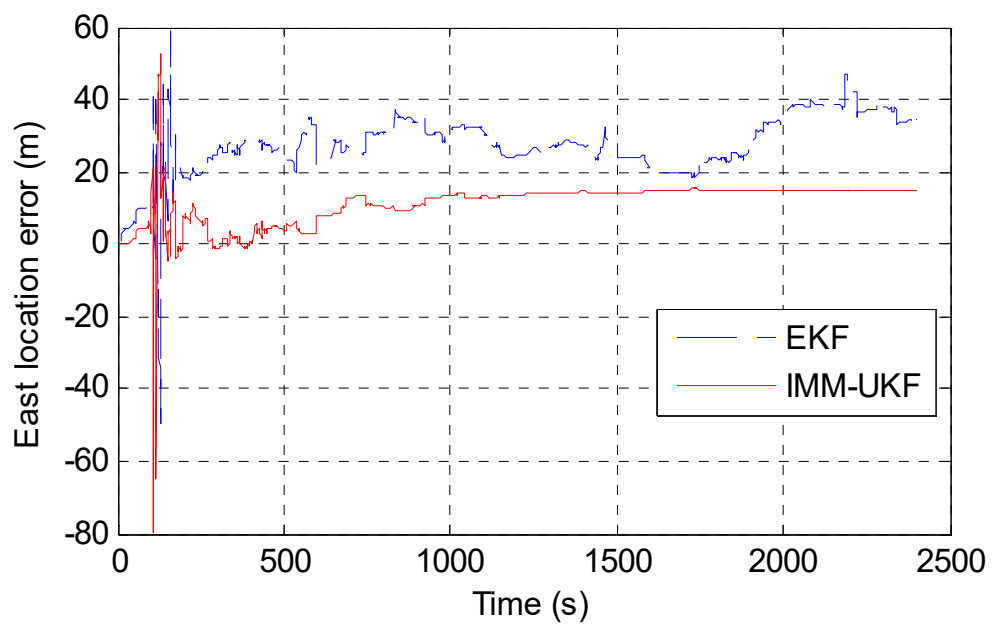

FIGURE 2. East position error

FIGURE 1. To format a figure caption, use the Microsoft Word template style: Figure Caption. The text "FIGURE 1," which labels the caption, should be bold and in upper case. If figures have more than one part, each part should be labeled (a), (b), etc.

Using a table, as in the above example, helps you control the layout 


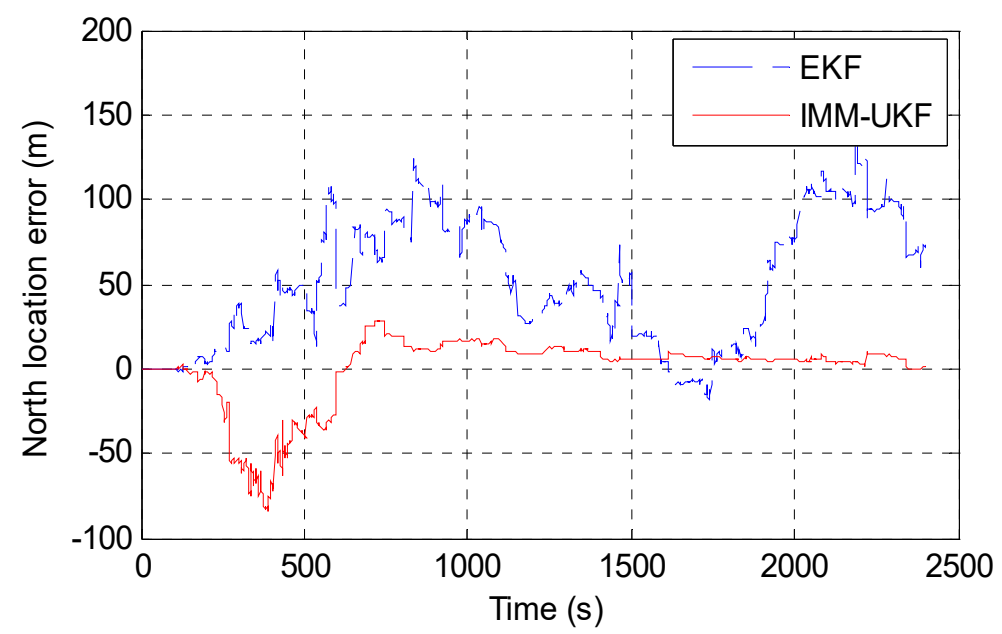

FIGURE 3. North position error

From the above simulation results, we can see that the accuracy of IMM-UKF algorithm is higher than that of traditional EKF algorithm under the same observation information. The proposed algorithm can effectively improve the accuracy of filter estimation. The high accuracy of IMM-UKF algorithm over EKF algorithm is that EKF algorithm introduces linearization error by linear first-order linearization of Taylor expansion of nonlinear function and reduces the estimation accuracy. For a general nonlinear system, the EKF algorithm cannot guarantee its convergence. Especially when there are multiple peaks in the probability distribution function, the error of the state estimation will be larger and even divergent. The UKF algorithm Unscented transform approximation of the actual probability distribution, there will be no linearization error, so its estimation accuracy is higher than the EKF method.

\section{CONCLUSION}

This paper mainly studies the civil UAV GPS failure under navigation problems. Adopted the second generation of Compass navigation systems for civil unmanned aerial vehicles. In order to avoid the problem of filtering divergence, linearization error and estimation error brought by navigation solution using standard IMM algorithm and EKF algorithm, an IMM-UKF nonlinear filtering algorithm is proposed to solve the navigation parameter of integrated navigation system And has carried on the simulation verification. The simulation results show that the proposed algorithm can improve the navigation accuracy and solve the problem of system nonlinearity and system noise uncertainty well.

\section{REFERENCES}

1. S. J. Julier, J. K. Uhlmann, H. F, Durrant. A new method for the nonlinear transformation of means and covariances in filters and estimators, IEEE Transactions on Automatic Control, 5(3): 477-482, 2000

2. Zhou J, Nie X, Lin J, A novel laser Doppler velocimeter and its integrated navigation system with strapdown inertial navigation, Optics \& Laser Technology, 64(6):319-323, 2014

3. Arifianto O, Farhood M, Optimal control of a small fixed-wing UAV about concatenated trajectories, Control Engineering Practice, 40:113-132, 2015

4. Gao J Z, Jia H G, Adaptive internal model control research in autonomous landing phase for a fixed-wing UAV, Ceas Aeronautical Journal, 8(1):45-51, 2017

5. Li Z, Wang J, Li B, et al, GPS/INS/Odometer Integrated System Using Fuzzy Neural Network for Land Vehicle Navigation Applications, Journal of Navigation, 67(6):967-983, 2014

6. Hyyti H, Visala A, A DCM Based Attitude Estimation Algorithm for Low-Cost MEMS IMUs, International Jouranl of Navigation and Observation, 2015(4):1-18, 2015 